\title{
Case report of metastatic presence of lingual lymph nodes in carcinoma alveolus of mandible
}

\author{
Jacob John ${ }^{1}$, Rahul Tiwari ${ }^{2, *}$, Kaushal Charan Pahari ${ }^{3}$, Philip Mathew ${ }^{4}$, Heena Tiwari ${ }^{5}$ \\ ${ }^{1,3} \mathrm{PG}$ Student, ${ }^{2}$ Fellow, ${ }^{4} \mathrm{HOD},{ }^{5} \mathrm{BDS},{ }^{1-4}$ Dept. of Oral \& Maxillofacial Surgery, ${ }^{1} \mathrm{KVG}$ Dental College and Hospital, Sullia, \\ Karnataka, ${ }^{2,4}$ Jubilee Mission Medical College \& Research Centre, Thrissur, Kerala, ${ }^{3}$ Surendra Dental College \& Research Institute, \\ Sriganga nagar, Rajasthan, ${ }^{5}$ Government Dental Surgeon, CHC, Makdi, Kondagaon, Chattisgarh, India
}

*Corresponding Author:

Email: drrahulvctiwari@gmail.com

\begin{abstract}
Lingual node metastasis is seen most commonly in carcinoma of tongue and carcinoma floor of mouth. Literature elicits about such cases, but rarely reported is lingual node metastasis in Squamous cell carcinoma of alveolus. Our case report is an elderly male patient with Squamous cell carcinoma of alveolus. Management included hemi-mandibulectomy because of the extension. Metastatic lingual node was found during the surgery as it is rare finding so is this case report presented to reappraise the literature.
\end{abstract}

Keywords: Squamous Cell Carcinoma, Neck Dissection, Lymphatics, Lingual Node, Metastasis.

\section{Introduction}

Carcinoma of oral cavity often metastasize to cervical lymph nodes extensive involvement of other lymph nodes makes the prognosis poor because the prognosis of oral cancer depends upon the control of the metastatic activity rather than the primary tumor removal of cervical lymph nodes by neck dissection including functional radical and modified are treatment of choice, recurrence rate often depends on the positive lymph nodes which are unintentionally left during the primary surgery. ${ }^{1-3}$ Usually lingual node are single metastatic nodes with no extra nodal invasion. Apart from the clinical methods of diagnosis, technology also plays a pivotal role. Imaging plays an important role in diagnosing cervical lymph nodes involvement which is a significant background workup of patients with head and neck cancer. ${ }^{4}$ The continuous advances in techniques have lead to the increased sensitivity of the imaging modalities in detection of lymph nodes. Various imaging techniques are used for the same which includes USG, Color Doppler USG, CT, CECT, MRI, PET and lymphoscintigraphy are most critical to diagnose the presence of lymph nodes in carcinoma. USG guided fine needle cytology is also preferred. ${ }^{5}$ It is known that lymph node occasionally located on lymph vessels of tongue are called as lingual lymph nodes. ${ }^{6}$ The existence of lingual lymph node had received little attention and no such studies of metastasis of oral cancer to these lymph nodes had been reported until Ozeki et al in 1985 first published his case report on patients with lymph node metastasis to lingual lymph nodes. Removal of lingual lymph nodes are not a part of regular neck dissection performed during the surgery, so is given little consideration. ${ }^{7,8}$ The anatomical data of these lymph nodes as well as their microscopic appearance may be of some importance in developing more effective surgical treatment for removal of these lymph nodes while operating oral cancer.

Case Report
A 62-year male was initially seen with a 3 months history of non-healing ulcer on gingivobuccal region of the mandibular right posterior region. Patient also presented with odynophagia. Clinical examination revealed a $2.7 \mathrm{~cm}$ non-healing ulcer present on gingival mucosa of second molar with palpable masses in the neck. Biopsy of the lesion area revealed squamous cell carcinoma with positive margins. With the help of biopsy report a computed tomography scan was planned to know the extend lesion, which demonstrated a tumor of right mandibular posterior alveolus with evidence of lymphadenopathy. TNM Staging was T2N2aM0. A right hemi mandibulectomy was performed through a lip split incision with continuity of modified radical neck decision. (Fig. 1) While resection of tumor an elastic hard right lateral lingual lymph node was palpated near the sublingual gland and send for histopathology. (Fig. 2)

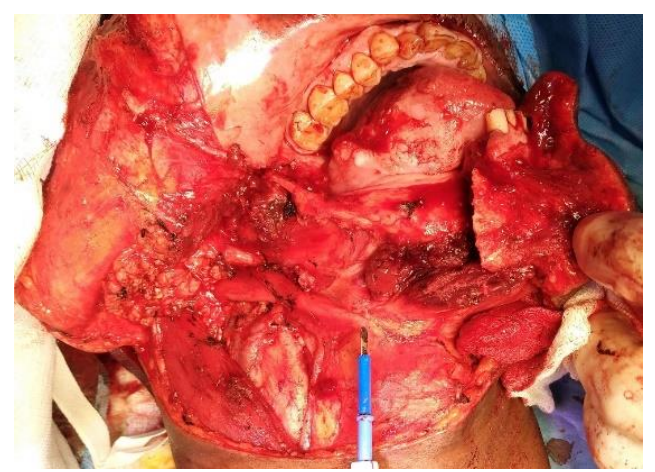

Fig. 1: Resection of the tumor (Hemi mandibulectomy) 


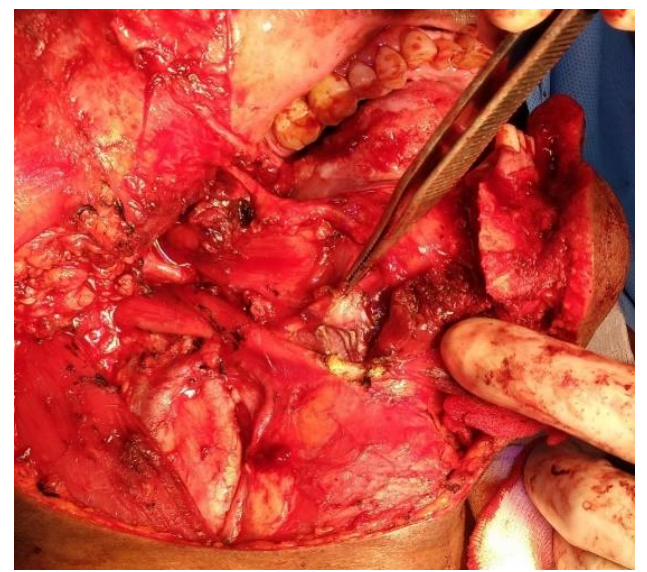

Fig. 2: Presence of Lingual Lymph Node

\section{Discussion}

There are various surgical approaches for removal of lingual lymph nodes discussed in the literature. The lingual lymph nodes are intermediate lymph nodes which are occasionally located outside the genioglossus muscle which is termed as lateral lingual lymph node or between the bilateral genioglossus muscle which are termed as median lymph nodes. Rouviere first noted the presence of the lingual lymph node and referred to them as the median and lateral lingual lymph node. ${ }^{9}$ Omura et al also identified the lingual lymph nodes as inconstant, interrupting nodules placed deeply above the suprahyoid region along the course of lymphatic vessels that drain the tongue and the floor of the mouth. ${ }^{10}$ However, according to the TNM classification, the lingual node is not classified as any level of necks, and there have been few reports of lingual node metastasis from oral cancer. Ozeki et al. first reported three cases of tongue cancer metastasizing to the lingual lymph nodes in $1985 .{ }^{11}$ Dutton et al. also reported on a patient with tongue cancer who had occult metastasis to the lingual lymph node and stated that a traditional discontinuous neck dissection may not remove all the lymph nodes at risk of metastasis from the oral cavity. ${ }^{12}$ However, the significance of this lymph node for cancer of the tongue and floor of the mouth has not been fully documented. Currently, the main causes of death from tongue cancer are now neck failure and distant metastasis. ${ }^{13}$ Neck recurrence of tongue cancer has been reported in the level II neck, especially in patients with multiple neck metastases associated with extra nodal invasion. ${ }^{14}$ However, such neck recurrence has rarely been observed recently, probably because of adequate dissection of the upper- and posterior part of the level II neck combined with resection of the posterior belly of the digastricus muscle and for advanced cases Para pharyngeal dissection of posterior invading tongue cancer. ${ }^{15}$ However, some of our patients showed neck recurrence near the hyoid bone. So, it is very important to promptly diagnose and plan accurate management to achieve a better prognosis. Such hindered lymph nodes must be removed.

Conclusion
This case offers further evidence that a traditional discontinuous neck dissection may not address all nodes at risk in oral cavity carcinoma. Further investigation for the lymph node distribution within the oral cavity is warranted to reappraise the upper limits of cervical lymphadenopathy. So, we recommend the policy that for each patient of head and neck cancer careful evaluation should be performed by means of various imaging modalities which will help to decide most appropriate treatment as well as reduced overall morbidity.

\section{References}

1. Goto. M., Hasegawa. Y., Terada. A., Hyodo. I., Hanai. N.et al. Prognostic significance of late cervical metastasis and distant metastasis in patients with stage I and II oral tongue cancers. Oral Oncol 41:62-9.

2. Godden. D.R., Ribeiro. N.F., Hassanein. K., Langton. S.G. Recurrent neck disease in oral cancer. J Oral Maxillofac Surg 60:748-53.

3. Umeda. M., Minamikawa. T., Yokoo. S., Komori. T. Metastasis of maxillary carcinoma to the parapharyngeal space: rationale and technique for concomitant en bloc parapharyngeal dissection. J Oral Maxillofac Surg 60:40813.

4. Umeda. M., Minamikawa. T., Komatsubara. H., Ojima. Y., Shibuya. Y., et al. T. En bloc resection of the primary tumour and cervical lymph nodes through the parapharyngeal space in patients with squamous cell carcinoma of the maxilla: a preliminary study. $\mathrm{Br} \mathrm{J} \mathrm{Oral}$ Maxillofac Surg 43:17-22.

5. Werner JA, D€unne AA, Myers JN. Functional anatomy of the lymphatic drainage system of the upper aerodigestive tract and its role in metastasis of squamous cell carcinoma. Head Neck 2003;25:322.

6. Huang SF, Kang CJ, Lin CY, et al. Neck treatment of patients with early stage oral tongue cancer: comparison between observation, supraomohyoid dissection, and extended dissection. Cancer 2008;112:1066.

7. D'Cruz AK, Vaish R, Kapre N, et al. Elective versus therapeutic neck dissection in node-negative oral cancer. $N$ Engl J Med 2015;373:521.

8. Yuen AP, Ho CM, Chow TL, et al. Prospective randomized study of selective neck dissection versus observation for N0 neck of early tongue carcinoma. Head Neck 2009;31:765.

9. Rouviere H. 1938. Anatomy of the human lymphatic system (Tobia MJ, editor and translator). Ann Arbor: Edward Brothers.

10. Omura K, Yanai C, Yamashita T. 1997. Diagnosis and management of lingual lymph node metastases. Int J Oral Maxillofac Surg 26:45-7.

11. Ozeki. S., Tashiro. H., Okamoto. M., Matsushima. T. 1985. Metastasis to the lingual lymph node in carcinoma of the tongue. J Maxillofac Surg 13:277-81.

12. Dutton, J.M., Graham, S.M., and Hoffman, H.T. 2002. Metastatic cancer to the floor of mouth: the lingual lymph nodes. Head Neck Surg 24:401-5.

13. Haberal I. Which is important in the evaluation of metastatic lymph nodes in head and neck cancer: palpation, ultrasonography, or computed tomography? Otolaryngol Head Neck Surg 2004;130:197.

14. Yuasa K, Kawazu T, Kunitake N, et al. Sonography for the detection of cervical lymph node metastases among patients with tongue cancer: criteria for early detection and assessment of follow-up examination intervals. Am J Neuroradiol 2000;21:1127. 
15. Hayashi T, Ito J, Taira $\mathrm{S}$, et al. The clinical significance of follow-up sonography in the detection of cervical lymph node metastases in patients with stage I or II squamous cell carcinoma of the tongue. Oral Surg Oral Med Oral Pathol Oral Radiol Endod 2003;96:112. 